Summary: Using the authors' previously developed method for the general kinetic analysis of non-linear irreversible polymerizations, the simulation of free radical homogeneous polymerization systems with terminal branching and chain transfer to polymer has been carried out for continuous stirred tank reactors. Its improved accuracy on the numerical evaluation of generating functions has been exploited in order to perform their numerical inversion and chain length distributions could also be estimated with or without the presence of gel. A comparison with alternative techniques emphasizing the effect of their simplifying assumptions on the accuracy of calculations is also presented.

Predicted CLD before gelation $(t=1 \mathrm{~h})$, after gelation ( $t=15 \mathrm{~h}$, steady state), and close to gel point for a free radical polymerization with transfer to polymer in a CSTR with $\tau=60 \mathrm{~min}$.

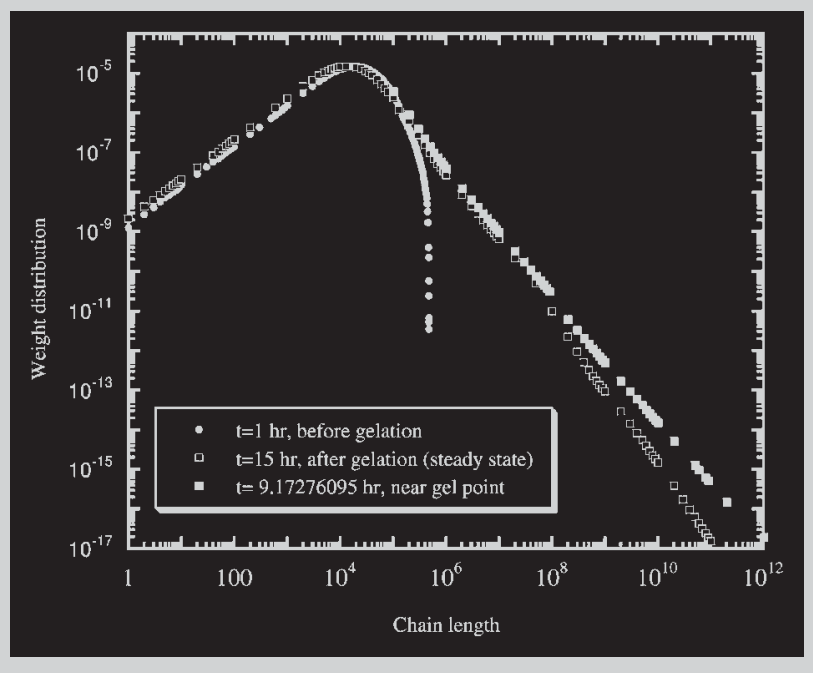

\title{
Transient Behavior and Gelation of Free Radical Polymerizations in Continuous Stirred Tank Reactors
}

\author{
Rolando C. S. Dias, ${ }^{1}$ Mário Rui P. F. N. Costa ${ }^{2}$ \\ ${ }^{1}$ LSRE, Instituto Politécnico de Bragança, Quinta de S. Apolónia, 5300 Bragança, Portugal \\ Fax: (+351) 273313051; E-mail: rdias@ipb.pt \\ ${ }^{2}$ LSRE-Faculdade de Engenharia da Universidade do Porto, Departamento de Engenharia Química, R. Roberto Frias, \\ 4200-465 Porto Codex, Portugal
}

Keywords: continuous stirred tank reactors; free radical polymerizations; gel; generating functions; numerical inversion; terminal branching; transfer to polymer

\section{Introduction}

The continuous production of polymers presents several advantages relatively to batch operation, particularly in free radical copolymerizations, as the operation in continuous stirred tank reactors (CSTR) offers the possibility of decreasing composition inhomogeneity. Therefore, many commercially important polymers are produced in CSTR. Styrene, ethylene, vinylidene chloride, acrylate and methacrylate esters and vinyl acetate, are some examples of monomers often polymerized using continuous processes. Unstirred tubular reactors, roughly equivalent to a cascade of CSTR with re-circulation, are also frequently used in these polymerization systems. A continuous backmix reactor ${ }^{[1]}$ would allow operation at high viscosities if needed.
Actually, a great number of polymerizations are carried out with emulsion processes, but in this paper we limit the analysis to the homogeneous systems, with the main emphasis on putting into evidence the effect of branching reactions in continuous systems, and the application of appropriate modeling techniques to its description.

In many free radical polymerizations, long chain branching can occur due to propagation on terminal double bonds and transfer to polymer. Ethylene, methyl acrylate, vinylidene chloride, and vinyl acetate polymerizations are some examples where branching is present as a consequence of these mechanisms. Vinyl acetate polymerization is one of the most representative systems in which terminal double bonds are created via transfer to monomer. Transfer to polymer via the reaction of chain radicals with active centers in the polymer is a common mode of branching in 
many free radical polymerizations. ${ }^{[2-4]}$ Different kinds of terminal double bonds, created through termination by disproportionation, can be formed with selectivities depending mainly on steric effects. ${ }^{[4]}$ As it is known since the 1950 s in the case of poly(methyl methacrylate), ${ }^{[5]}$ they can react with polymer radicals leading to block copolymers, but the impact of these reactions in chain length distributions (CLD) should usually be minor as discussed below.

It is well known that free radical polymerizations are kinetically controlled and therefore the condition for random formation of branch points in a statistical gelation process cannot be applied. This fact invalidates the mathematical treatment of this systems by the theory of branching processes. ${ }^{[6]}$ A possible exception are chemical systems (such as in polyethylene formation) in which radical breakage can "shuffle" enough CLD, but there is so far no confirmation of this hypothesis.

Modeling non-linear free radical polymerizations in CSTR has been for a long time a somewhat controversial subject. Based on Bamford and Tompa's pioneering work, ${ }^{[7]}$ Nagasubramanian and Graessley ${ }^{[8]}$ formulated a model for application to the solution vinyl acetate polymerization in a CSTR. Their main objective was to make an extension from previous experimental and theoretical studies in batch reactors ${ }^{[9-12]}$ to continuous operation. These models were derived by writing population balances of polymer species in the reactor and a set of ordinary differential equations results for the moments of the CLD.

When the comparison between the simulations obtained with this kind of models with experimental results ${ }^{[13]}$ was carried out, two major difficulties were identified: divergence of the second moment (gelation) was predicted but not observed and it was not possible to simultaneously predict the behavior of batch and CSTR reactors with a unified set of kinetic parameters.

These discrepancies have originated new efforts in developing simulation methods and experimental studies of the continuous polymerization of vinyl acetate carried out by this same group. Some attempts in the inclusion of initiation, termination, and inhibition effects in the models have also not been well succeeded. ${ }^{[14]}$ Other sets of experimental data were obtained in batch and continuous reactors with especial care about the elimination of inhibition periods, ${ }^{[15,16]}$ but the same inconsistencies above identified between model predictions and experimental observations have remained.

In other works, the polymerization of vinyl acetate was done in different experimental conditions ${ }^{[17]}$ (higher polymerization rates) but on comparing the experimental results obtained with an improved version of previous developed models, no satisfactory agreement was achieved. ${ }^{[18]}$

A significant part of the above mentioned discrepancies between experimental observations and model predictions can be explained by the approximations introduced in the calculations. In order to obtain a tractable model, some of the simplifying assumptions given below are systematically found in those works:

A1) Quasi-steady state for radical concentrations.

A2) Negligible rates of initiation and termination reactions relatively to propagation and transfer.

A3) Negligible presence of multiple radical centers.

A4) Negligible presence of more than one terminal double bond per molecule.

A5) Negligible exit of radicals from the reactor.

A6) Chain transfer only to "dead" polymer.

A7) Closure conditions for the moments.

More than one decade ago, Arriola ${ }^{[19]}$ has shown that in the modeling of addition polymerization systems, assumptions A1-A7 can all be simultaneously eliminated if all polymer molecules containing free radical sites or devoid of them ("dead" species) are assumed to engage in the same reactions.

Nevertheless, in later published related works using the method of the moments, ${ }^{[20,21]}$ less exact models have been used, probably in order to save computational resources. Due to similar difficulties, alternative techniques for modeling non-linear free radical polymerizations have also introduced some of the aforementioned approximating conditions. Such is the case of simulations of free radical polymerizations using "numerical fractionation", [22,23] Mote Carlo method, ${ }^{[2,25]}$ and Galerkin finite-elements method. ${ }^{[26,27]}$ The problems to be faced in the modeling of this kind of systems become much tougher if gel is present and if the transient behavior of the reactor is to be described. Therefore some further limitations in the application of these modeling techniques can also be identified:

L1) Analysis restricted to the steady state operation of the reactor

L2) Analysis restricted to the pre-gel region.

In Table 1 the approximation conditions and the limitations of these approaches are summarized.

While this paper was being prepared, Iedema and Hoefsloot ${ }^{[28]}$ have published an analysis of the role of poly-radicals in the gelation when transfer to polymer is present and have accordingly managed to adapt the Galerkin finite element method ${ }^{[29,30]}$ they had been using. A key improvement was the consideration that all polymer

Table 1. Approximation conditions and limitations of alternative techniques for the modeling of non-linear free radical polymerizations.

\begin{tabular}{ll}
\hline Method & $\begin{array}{c}\text { Approximation conditions } \\
\text { and limitations }\end{array}$ \\
\hline Method of the moments $^{[19]}$ & L2 \\
Method of the moments $^{[20]}$ & A3, L2 \\
Method of the moments $^{[21]}$ & A3, A4, A6, A7, L2 \\
Numerical fractionation $^{[22,23]}$ & A1, A3, A4, A5, A6, A7 \\
Monte Carlo method $^{[24,25]}$ & A2, L1, L2 \\
Galerkin finite-elements method $^{[26]}$ & A3, A6, L2
\end{tabular}


molecules, regardless of the presence of free radical sites, could participate in the same propagation and transfer reactions, eliminating the main source of inconsistencies in the rate equations. A few approximations were due to limitations of the numerical method as implemented in the commercial software used (such as assuming a maximum number of radical sites or chain length in sol), but the impact of these in the accuracy of predictions is likely to be small, and should become negligible if it were possible to increase these values as needed.

Calculations in real chain length domain are too slow if a "brute force" way of solving mass balance equations for all species is chosen. The problem lies in the convolution sums in the terms describing, e.g., propagation on pendent double bonds and termination by combination. It is nevertheless possible to use interpolation along chain length in order to save computer time. ${ }^{[29,30]}$ This approach has mainly been used with 1-D distributions, but it certainly could be generalized to multi-dimensional distributions at the price of a correspondent increased memory expenditure.

If it is acceptable to describe termination reactions using appropriate average values (chain length independent) of apparent rate constants, approaches based on Laplace domain analysis become very competitive in computing efficiency as far as prediction of average values over CLD are concerned.

The interest of improving mathematical accuracy in kinetic modeling of non-linear free radical polymerizations has already been stressed long time ago by Kuchanov and Pis'men, ${ }^{[31]}$ namely the need for avoiding quasi-steady state hypothesis for radical concentrations and the consideration of multiple radical centers. It is not straightforward to satisfy both these conditions.

The authors have introduced some improvements into a more rigorous kinetic approach for modeling irreversible non-linear polymerizations. ${ }^{[32,33]}$ This method can be used for the prediction of average molecular weights and molecular weight distributions before and after gelation, and elastic properties of the gel can also be calculated. An extension to the prediction of average radius of gyration of branched polymers in non-radical poly-additions ${ }^{[34]}$ has been introduced.

Nevertheless, and due to the lack of suitable numerical methods, only very recently it became possible to extend this method to the post-gelation region in non-linear free radical polymerizations, such as free radical copolymerizations of mono/divinyl monomers. ${ }^{[35]}$ Non-linear free radical polymerizations with terminal branching and chain transfer to polymer in batch reactors have also been studied using this approach, ${ }^{[36]}$ and the influence of the different branching reactions on the average molecular weights before and after gelation has also been put into evidence.

The main goal of this paper is to extend our previous analysis for batch reactors to the CSTR operation showing that the quantification of gelation in continuous reactors avoiding all approximation conditions A1-A7 is possible. At the same time, limitations L1 and L2 are overcome and therefore improved theoretical predictions are obtained.

A kinetic scheme as generic as possible is chosen in order to find common patterns of behavior of similar chemical systems. A brief analysis of a system with kinetics close to the usually accepted for the vinyl acetate polymerization (with no attempt of claiming an exact description of it) is also carried out. The general principles of this method have been presented and discussed elsewhere ${ }^{[37]}$ and so, in this work, only the main steps of its application to this particular system need to be shown.

\section{Kinetic Scheme}

The most general kinetic scheme considered in this work comprises of the following steps:

- Initiator decomposition.

- Initiation of monomer and terminal double bonds by primary radicals from the initiator and from the solvent.

- Propagation of monomer and terminal double bonds with creation of active centers for transfer to polymer.

- Termination by combination.

- Termination by disproportionation with formation of terminal double bonds (TDBD).

- Transfer to polymer.

- Transfer to monomer with formation of terminal double bonds (TDBM).

- Transfer to solvent.

Terminal double bonds, created in termination by disproportionation, are distinguished from the terminal double bonds originated in the transfer to monomer reaction because they should have very different reactivities, as already considered in a previous model for vinyl acetate polymerization. ${ }^{[27]}$ The latter are chemically similar to the double bonds in monomer, but they should have a somewhat lower reactivity owing to increased steric hindrance. Unsaturated terminal groups in polymethacrylates add free radicals, but instead of propagating they mostly undergo $\beta$ chain-scission $^{[38]}$ so they behave mostly as sites for chain transfer, without much influence in CLD in usual conditions ${ }^{[39]}$ due to their low concentration.

This scheme is consistent with NMR studies of vinyl acetate and butyl acrylate free radical polymerizations. ${ }^{[40,41]}$

There is a total number of $N_{\mathrm{A}}=12$ groups $\mathrm{A}_{j}, j=$ $1, \ldots, 12$ :

$A_{1}$ : A lumped set of different kinds of free radicals (Radical from the monomer and radical in a side chain of the monomer, for example).

$A_{2}$ : Terminal double bonds originated by transfer to monomer.

$A_{3}$ : Terminal double bonds originated by termination by disproportionation. 
$A_{4}$ : Site of transfer to polymer.

$A_{5}$ : Monomer.

$A_{6}$ : Solvent.

$A_{7}$ : Primary radicals from the initiator.

$A_{8}$ : Primary radicals from the solvent.

$A_{9}$ : Initiator.

$A_{10}$ : Polymerized monomer unit.

$A_{11}$ : Fragment from initiator.

$A_{12}$ : Fragment from solvent.

Seven of these groups belong to the polymer, $N_{\mathrm{P}}=7$ (groups with $\delta_{\mathrm{P}_{j}}=1$, otherwise $\delta_{\mathrm{P}_{j}}=0$ ). Nine groups are active (groups with $\delta_{\mathrm{A}_{j}}=1$, otherwise $\delta_{\mathrm{A}_{j}}=0$ ) and the first four are present in the polymer, $N_{\mathrm{A}_{\mathrm{p}}}=4$. Five groups are active but they are not present in the polymer $\left(\delta_{\mathbf{P}_{j}}=0\right)$. This description is presented with more detail in Table 2.

Groups corresponding to the fragments from the initiator and solvent can be eliminated, if only average monomer chain lengths are needed.

This kinetic scheme considers 15 elementary reactions (see Table 3), which can be divided into 5 different classes. A total number of $N_{\mathrm{R}}=4$ reactions create connections between repeating units and of these $N_{\mathrm{R}_{\mathrm{p}}}=3$ involve only groups present in polymer molecules (propagation of terminal double bonds and termination by combination) and $N_{\mathrm{R}}-N_{\mathrm{R}_{\mathrm{P}}}=1$ involve a group in the polymer and a non-polymeric molecule (propagation of monomer). All these reactions generate sites of transfer to polymer. A total number of $N_{\mathrm{R}}^{* *}=7$ bimolecular reactions do not create connections between repeating units, $N_{\mathrm{R}_{\mathrm{p}}}^{* *}=2$ of these involve two polymer molecules (transfer to polymer and termination by disproportionation) and $N_{\mathrm{R}_{\mathrm{S}}}^{* *}=5$ involve a polymer molecule and a small molecule (terminal double bonds initiation and transfer to solvent). The other reactions are $N_{\mathrm{R}}^{*}=1$ unimolecular reactions (initiator decomposition), $N_{\mathrm{I}}=2$ bimolecular reactions involving groups not present in the polymer with polymer creation (monomer initiation by primary radicals from initiator and solvent) and $N_{\mathrm{M}}=1$ are transfers to monomer also leading to polymer formation.

As the main goal of this presentation is to show how the usual set of simplifying conditions in modeling nonlinear free radical polymerizations in CSTR can have an important effect on model predictions, we have decided to use as much as possible previous kinetic schemes without introducing new chemical species and rate parameters. So, we are going to try to isolate some consistency problems of mathematical modeling with a rather over-simplified kinetic scheme.

\section{Prediction of Moment Generating Function of Chain Length Distribution and Average Molecular Weights}

The set of polymer species with same number of groups, described by vector $\mathbf{a}_{m}$, is named $\mathrm{P}\left(\mathbf{a}_{m}\right)$ and its corresponding concentration is $P\left(\mathbf{a}_{m}\right)$. This approach aims at obtaining the vectorial moment generating function:

$$
G(\mathbf{s})=\sum_{\mathrm{a}_{1}=0}^{\infty} \ldots \sum_{\mathrm{a}_{N_{\mathrm{A}}}=0}^{\infty} s_{1}^{\mathrm{a}_{1}} \ldots s_{N_{\mathrm{A}}}^{\mathrm{a}_{N_{\mathrm{A}}}} \mathrm{P}\left(\mathrm{a}_{1}, \ldots, \mathrm{a}_{N_{\mathrm{A}}}\right)
$$

The system discussed here consist of $N_{\mathrm{A}}-N_{\mathrm{p}}=5$ groups which are chemically active but not present in the polymer $\left(\delta_{\mathrm{P}_{i}}=0\right)$. The number of components of vectors $\mathbf{a}$ and $\mathbf{s}$, as well as the number of sums in Equation (1) are only $N_{\mathrm{P}}$ in the computational implementation. So, $\mathrm{P}\left(\mathrm{a}_{1}, \ldots, \mathrm{a}_{N_{\mathrm{A}}}\right)=0$ whenever, at least one of the counts of groups $\mathrm{a}_{j}$ not belonging to polymer $\left(\delta_{\mathrm{P}_{j}}=0\right)$ is not nil, and in the definition Equation (1) the corresponding Laplace parameters, $s_{j}$, should be set equal to 1 .

Following the methods we have already presented, ${ }^{[32,37]}$ it is possible to write the generating function of the rate equations of formation of polymer species by chemical reaction. For a CSTR with fixed reaction volume and constant space-time $\left(\tau=\frac{V}{Q}\right)$, the mass balance equation in

Table 2. Description of groups considered in a free radical polymerization with terminal double bonds branching and transfer to polymer in a continuous stirred tank reactor (CSTR).

\begin{tabular}{|c|c|c|c|c|}
\hline Group description & $j$ & $\delta_{\mathrm{P}_{j}}$ & $\delta_{\mathrm{A}_{j}}$ & $\begin{array}{l}\text { Chemical formula } \\
\text { (example) }\end{array}$ \\
\hline Free radical & 1 & 1 & 1 & $-\mathrm{HYC}^{\bullet}$ \\
\hline Terminal double bond originated by transfer to monomer (TDBM) & 2 & 1 & 1 & $\mathrm{CH}_{2}=\mathrm{CH}-$ \\
\hline Terminal double bond originated by termination by disproportionation (TDBD) & 3 & 1 & 1 & $\mathrm{CHY}=\mathrm{CH}-$ \\
\hline Transfer to polymer center & 4 & 1 & 1 & $-\mathrm{CH}_{2}-\mathrm{C} \underline{H} \mathrm{Y}-\mathrm{CH}_{2}-$ \\
\hline Monomer & 5 & 0 & 1 & $\mathrm{CH}_{2}=\mathrm{CHY}$ \\
\hline Solvent & 6 & 0 & 1 & $\mathrm{C}\left(\mathrm{CH}_{3}\right)_{3} \mathrm{OH}$ \\
\hline Primary radical from the initiator & 7 & 0 & 1 & $\left(\mathrm{CH}_{3}\right)_{2} \mathrm{CNC}^{\bullet}$ \\
\hline Primary radical from the solvent & 8 & 0 & 1 & $\left(\mathrm{CH}_{3}\right)_{2} \mathrm{C}(\mathrm{OH}) \mathrm{H}_{2} \mathrm{C}^{\bullet}$ \\
\hline Initiator & 9 & 0 & 1 & $\left(\mathrm{CH}_{3}\right)_{2} \mathrm{CNCN}=\mathrm{NCCN}\left(\mathrm{CH}_{3}\right)_{2}$ \\
\hline Polymerized monomer unit & 10 & 1 & 0 & $-\mathrm{CH}_{2}-\mathrm{CHY}-$ \\
\hline Initiator fragment & 11 & 1 & 0 & $\left(\mathrm{CH}_{3}\right)_{2} \mathrm{CNC}-$ \\
\hline Solvent fragment & 12 & 1 & 0 & $\left(\mathrm{CH}_{3}\right)_{2} \mathrm{C}(\mathrm{OH}) \mathrm{H}_{2} \mathrm{C}-$ \\
\hline
\end{tabular}


Table 3. Reaction scheme in a free radical polymerization with terminal double bonds branching and transfer to polymer in a CSTR.

\begin{tabular}{|c|c|}
\hline Reaction name & Chemical equation \\
\hline Initiator decomposition & $\mathrm{A}_{9} \stackrel{\mathrm{k}_{1}^{*}=\mathrm{k}_{\mathrm{d}}}{\longrightarrow} 2 f \mathrm{~A}_{7}$ \\
\hline Monomer initiation by primary radicals from initiator & $\mathrm{A}_{5}+\mathrm{A}_{7} \stackrel{\mathrm{k}_{\mathrm{I}_{1}}=\mathrm{k}_{\mathrm{i}_{1}}}{\longrightarrow} \mathrm{A}_{1}+\mathrm{A}_{10}+\mathrm{A}_{11}$ \\
\hline Monomer initiation by primary radicals from solvent & $\mathrm{A}_{5}+\mathrm{A}_{8} \stackrel{\mathrm{k}_{\mathrm{I}_{2}}=\mathrm{k}_{\mathrm{i}_{1 \mathrm{~s}}}}{\longrightarrow} \mathrm{A}_{1}+\mathrm{A}_{10}+\mathrm{A}_{12}$ \\
\hline Propagation of TDBM & $\mathrm{A}_{1}+\mathrm{A}_{2} \stackrel{\mathrm{k}_{1}=\mathrm{kp}_{\mathrm{p}}}{\longrightarrow} \mathrm{A}_{4}+\mathrm{A}_{1}$ \\
\hline Propagation of TDBD & $\mathrm{A}_{1}+\mathrm{A}_{3} \stackrel{\mathrm{K}_{2}=\mathrm{k}_{\mathrm{p}_{3}}}{\longrightarrow} \mathrm{A}_{4}+\mathrm{A}_{1}$ \\
\hline Termination by combination & $\mathrm{A}_{1}+\mathrm{A}_{1} \underset{\mathrm{k}_{1}=\mathrm{k}}{\stackrel{\mathrm{k}_{3}=\mathrm{k}_{\mathrm{tc}}}{\longrightarrow}} \mathrm{A}_{4}+\mathrm{A}_{4}$ \\
\hline Propagation of monomer & $\mathrm{A}_{1}+\mathrm{A}_{5} \stackrel{\mathrm{k}_{4}=\mathrm{k}_{\mathrm{p}_{1}}}{\longrightarrow} \mathrm{A}_{4}+\mathrm{A}_{1}+\mathrm{A}_{10}$ \\
\hline Transfer to polymer & $\mathrm{A}_{1}+\mathrm{A}_{4} \stackrel{\mathrm{K}_{1}=\mathrm{K}_{\mathrm{fp}}}{\longrightarrow} \mathrm{A}_{1}$ \\
\hline Termination by disproportionation & $\mathrm{A}_{1}+\mathrm{A}_{1} \stackrel{\mathrm{k}_{2}^{* *}=\mathrm{k}_{\mathrm{td}}}{\longrightarrow} \mathrm{A}_{3}$ \\
\hline Initiation of TDBM by primary radical from initiator & $\mathrm{A}_{2}+\mathrm{A}_{7} \stackrel{\mathrm{k}_{3}^{* *}=\mathrm{k}_{\mathrm{i}_{2}}}{\longrightarrow} \mathrm{A}_{1}+\mathrm{A}_{11}$ \\
\hline Initiation of TDBM by primary radical from solvent & $\mathrm{A}_{2}+\mathrm{A}_{8} \underset{\mathrm{k}_{4}^{* *}-\mathrm{k}_{\mathrm{k}_{25}}}{\longrightarrow} \mathrm{A}_{1}+\mathrm{A}_{12}$ \\
\hline Initiation of TDBD by primary radical from initiator & $\mathrm{A}_{3}+\mathrm{A}_{7} \underset{\mathrm{k}_{5 *-1}=\mathrm{K}_{\mathrm{i}_{3}}}{\longrightarrow} \mathrm{A}_{1}+\mathrm{A}_{11}$ \\
\hline Initiation of TDBD by primary radical from solvent & $\mathrm{A}_{3}+\mathrm{A}_{8} \stackrel{\mathrm{k}_{6}^{* *}=\mathrm{k}_{\mathrm{i}_{3 \mathrm{~s}}}}{\longrightarrow} \mathrm{A}_{1}+\mathrm{A}_{12}$ \\
\hline Transfer to solvent & $\mathrm{A}_{1}+\mathrm{A}_{6} \stackrel{\mathrm{k}_{7}^{* *}=\mathrm{k}_{\mathrm{s}}}{\longrightarrow} \mathrm{A}_{8}$ \\
\hline Transfer to monomer & $\mathrm{A}_{1}+\mathrm{A}_{5} \stackrel{\mathrm{k}_{\mathrm{M} 1}=\mathrm{k}_{\mathrm{fm}}}{\longrightarrow} \mathrm{A}_{1}+\mathrm{A}_{2}+\mathrm{A}_{10}$ \\
\hline
\end{tabular}

Laplace domain describing this polymerization scheme is as follows:

$$
\begin{aligned}
\frac{\partial G}{\partial t}= & k_{p_{2}}\left(\frac{s_{4}}{s_{2}} \frac{\partial G}{\partial \log s_{1}} \frac{\partial G}{\partial \log s_{2}}-A_{2} \frac{\partial G}{\partial \log s_{1}}-A_{1} \frac{\partial G}{\partial \log s_{2}}\right) \\
& +\mathrm{k}_{\mathrm{p}_{3}}\left(\frac{s_{4}}{s_{3}} \frac{\partial G}{\partial \log s_{1}} \frac{\partial G}{\partial \log s_{3}}-\mathrm{A}_{3} \frac{\partial G}{\partial \log s_{1}}-\mathrm{A}_{1} \frac{\partial G}{\partial \log s_{3}}\right) \\
& +\mathrm{k}_{\mathrm{tc}}\left[\frac{s_{4}^{2}}{s_{1}^{2}}\left(\frac{\partial G}{\partial \log s_{1}}\right)^{2}-2 \mathrm{~A}_{1} \frac{\partial G}{\partial \log s_{1}}\right] \\
& +k_{p_{1}} A_{5} \frac{\partial G}{\partial \log s_{1}}\left(s_{4} s_{10}-1\right) \\
& +\mathrm{k}_{\mathrm{fp}}\left[\mathrm{A}_{4} \frac{\partial G}{\partial \log s_{1}}\left(\frac{1}{s_{1}}-1\right)+\mathrm{A}_{1} \frac{\partial G}{\partial \log s_{4}}\left(\frac{s_{1}}{s_{4}}-1\right)\right] \\
& +\mathrm{k}_{\mathrm{td}}\left[\mathrm{A}_{1} \frac{\partial G}{\partial \log s_{1}}\left(\frac{1}{s_{1}}-1\right)+\mathrm{A}_{1} \frac{\partial G}{\partial \log s_{1}}\left(\frac{s_{3}}{s_{1}}-1\right)\right] \\
& +\mathrm{k}_{\mathrm{i}_{2}} \mathrm{~A}_{7} \frac{\partial G}{\partial \log s_{2}}\left(\frac{s_{1} s_{11}}{s_{2}}-1\right)+\mathrm{k}_{\mathrm{i}_{2 \mathrm{~s}}} \mathrm{~A}_{8} \frac{\partial G}{\partial \log s_{2}}\left(\frac{s_{1} s_{12}}{s_{2}}-1\right) \\
& +\mathrm{k}_{\mathrm{i}_{3}} \mathrm{~A}_{7} \frac{\partial G}{\partial \log s_{3}}\left(\frac{s_{1} s_{11}}{s_{3}}-1\right)+\mathrm{k}_{\mathrm{i}_{\mathrm{s}}} \mathrm{A}_{8} \frac{\partial G}{\partial \log s_{3}}\left(\frac{s_{1} s_{12}}{s_{3}}-1\right) \\
& +\mathrm{k}_{\mathrm{s}} \mathrm{A}_{6} \frac{\partial G}{\partial \log s_{1}}\left(\frac{1}{s_{1}}-1\right) \\
& +\mathrm{k}_{\mathrm{fm}} \mathrm{A}_{5}\left[\mathrm{~A}_{1} s_{1} s_{2}+\frac{\partial G}{\partial \log s_{1}}\left(\frac{1}{s_{1}}-1\right)\right] \\
& +\mathrm{k}_{\mathrm{i}_{1}} \mathrm{~A}_{5} \mathrm{~A}_{7} s_{1} s_{10} s_{11}+\mathrm{k}_{\mathrm{i}_{1 \mathrm{~s}}} \mathrm{~A}_{5} \mathrm{~A}_{8} s_{1} s_{10} s_{12}+\frac{G_{F}-G}{\tau}
\end{aligned}
$$

The initial value $\mathbf{s}_{0}$ of the vector of Laplace parameters $\mathbf{S}$ has to be computed as a function of its final value, $\mathbf{s}_{f}$, which is prescribed for a certain time of polymerization $t=t_{f}$ as before presented. ${ }^{[35-37]}$ In order to carry out the prediction of the CLD some component of the vector $\mathbf{s}_{f}$, let us say $\mathrm{s}_{\mathrm{k}_{f}}$, admit a set of complex or real numbers with $\left|s_{\mathrm{k}_{f}}\right| \leq 1$ as will be discussed later.

More often, the goal is only to compute the moments of the CLD and therefore $\mathbf{s}_{f}=1$. A possible solution is $\mathbf{s}_{0}=1$. This trivial solution is the physically meaningful solution before gelation (all groups belong to finite polymer molecules). Gel point is a bifurcation point where a second branch (the non-trivial solution ) with at least some $\mathrm{s}_{j_{0}}<1$, intersects the trivial solution for $t=t_{\mathrm{g}}$, the gel time. If we are only interested in the average properties of the polymer before gelation the problem is relatively simple and a system of ordinary differential equations must be solved as an initial conditions problem.

Kinetic parameters for these case studies are presented in Table 4. The propagation rate constant for the monomer is a recently measured value by PLP for vinyl acetate ${ }^{[42]}$ and the values for ratios of constants relative to transfer to monomer, transfer to polymer, transfer to solvent, and propagation of terminal double bonds were taken from earlier works also dealing with vinyl acetate polymerization. ${ }^{[10,11,13]}$ Termination is supposed to occur with equal probability by combination and by dismutation.

It is known that in most polymerizations the values of the chain termination rate coefficients, initiator efficiency, or even the propagation rate coefficients can decrease significantly when polymer concentration increases. These dependencies can be inserted in the set of differential equations by expressing rate coefficients as functions of monomer conversion, excess Van der Waals volume fraction or other kind of correlation. Since the main objective of this work is to test the ability of the presented method to deal with branching for polymerization in CSTR with an 
Table 4. Representative set of kinetic parameters in a free radical polymerization with terminal double bonds branching and transfer to polymer in a CSTR (system I).

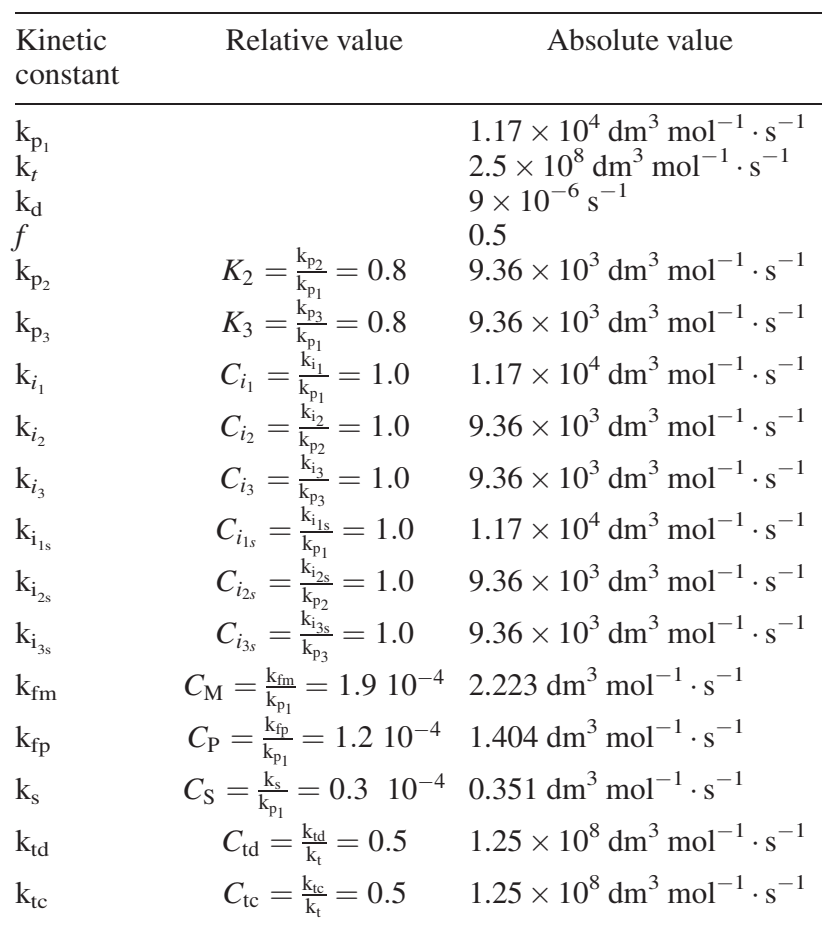

improved mathematical exactness, isothermal reactors and constant rate coefficients are assumed.

Initial and feed concentrations of monomer, solvent, and initiator used in the simulations are typical values present in experimental works in this subject ${ }^{[13,16]}$ and are reported in Table 5. Molecular masses of monomer, solvent and initiator fragments were taken as the corresponding values of 86,73 , and 68 for vinyl acetate, $t$-butanol, and AIBN, respectively. In these conditions with typical values of space-time in the order of $\tau=1-10 \mathrm{~h}$, steady state monomer conversions of $0.15-0.60$ can be obtained.

As it happened with batch reaction, ${ }^{[36]}$ the first step in simulations is the calculation of gel time, by integration of the equations for the second order moments along the trivial solution branch until divergence to infinity. This initial value problem presents no difficulties. Average molecular weights before gelation are also obtained by this procedure. In Figure 1, the predicted dimensionless gel time $\left(\theta_{\mathrm{g}}=\frac{t_{\mathrm{g}}}{\tau}\right)$ as a function of the space-time for three different molar ratios between solvent and monomer $\left(y_{\mathrm{S}}=\frac{\mathrm{S}_{0}}{\mathrm{M}_{0}}\right)$ are plotted.

Table 5. Initial and feed concentrations.

\begin{tabular}{|c|c|c|c|}
\hline $\operatorname{Monomer}\left(M_{0}\right)$ & Solvent $\left(S_{0}\right)$ & Initiator $\left(I_{0}\right)$ & Ratio between \\
\hline $\mathrm{mol} \cdot \mathrm{dm}^{-3}$ & $\mathrm{~mol} \cdot \mathrm{dm}^{-3}$ & $\mathrm{~mol} \cdot \mathrm{dm}^{-3}$ & $\begin{array}{l}\text { solvent and } \\
\text { monomer }\left(y_{\mathrm{s}}\right)\end{array}$ \\
\hline 3.57 & 7.14 & $10^{-3}$ & 2 \\
\hline 2.14 & 8.56 & $10^{-3}$ & 4 \\
\hline 1.52 & 9.12 & $10^{-3}$ & 6 \\
\hline
\end{tabular}

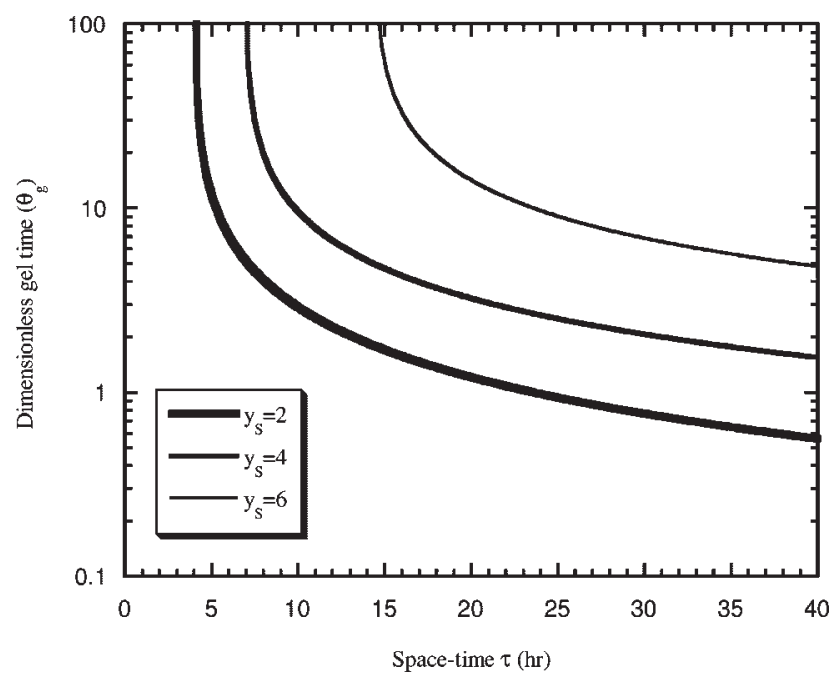

Figure 1. Dimensionless gel time $\theta_{\mathrm{g}}$ in a continuous stirred tank reactors (CSTR) as a function of the operation space-time $\tau$.

As expected, gel time increases with growing solvent concentration due to chain transfer to solvent. Note that monomer dilution also contributes to the decrease of importance of transfer to polymer and, thus to the increased gel time. Critical values of space-time of 4.07, 7.01, and $14.51 \mathrm{~h}$ are identified for $y_{\mathrm{s}}=2,4$, and 6 , respectively. Below these critical values, in each case, no gelation occurs.

After gelation, a vector of final times (in each case $t_{f}>t_{\mathrm{g}}$ ) is chosen in order to obtain the properties of the polymer at that time. The method is analogous to the described in a previous paper ${ }^{[36]}$ and only the few small differences found are worth mentioning. Characteristic lines with a CSTR show a smoother behavior than with batch reactors, specially for long final times.

All computations presented in this work have been carried out in a workstation with a double Intel Xeon $2.8 \mathrm{GHz}$ processor with $4 \mathrm{~GB}$ memory, running Red Hat Linux 9.0 (kernel version 2.4.20-8). Fortran sources were compiled using GNU compiler g77 based on gcc 3.2.2. Typically, the CPU time spent to solve each problem of characteristics is in the order of $377 \mathrm{~s}$.

Prediction of average molecular weights has been done in a way similar to batch reaction and also presents very different levels of difficulty depending on whether gel is present or not. Before gelation, it reduces to an initial value problem with $N_{\mathrm{A}}+N_{\mathrm{P}}+\frac{N_{\mathrm{P}}\left(N_{\mathrm{P}}+1\right)}{2}+1=48$ equation to obtain moments up to second order, easily solved using, for instance, the integrator RADAU5 ${ }^{[43]}$ The whole computation of average molecular weights before gelation is done with a single integration in which the gel point is identified by the divergence of the second order moments. For the current chemical systems discussed here, this required CPU times of about $6 \mathrm{~s}$. Local relative tolerance in RADAU5 has been varied between $10^{-8}$ and $10^{-12}$, while absolute tolerance was kept fixed at a value of $10^{-16}$, which is limited by 
machine precision. Numerical results have agreed with at least 8 decimal places. The same range of tolerances was used in the simulations with "numerical fractionation" technique and method of the moments to be presented later in this work.

After gelation, for each prescribed time in which the average molecular weights of the polymer are to be computed, an independent problem must be solved. Typically, in this chemical system, a single point in the post-gel region requires $380 \mathrm{~s}$ of CPU time (and 14\% of memory). Thus, getting the solution for the characteristics is the controlling step in terms of CPU time in the post-gel region.

There is only a compound tolerance parameter for code $\mathrm{ACDC},{ }^{[44]}$ which has been varied between $10^{-6}$ and $10^{-8}$, and numerical results agree to 8 decimal places.

In Figure 2, results for the unsteady state operation of a CSTR with $\tau=8 \mathrm{~h}$ in the pre- and post-gel regions are shown. With these operating conditions a dimensionless time for gelation of $\theta_{\mathrm{g}}=4.09$ is predicted. It should be noticed that the time needed for reaching steady state in terms of monomer conversion and average molecular weights, or sol fraction are very different. $\theta=2$ is enough to reach the steady state in monomer concentration, whereas a value around $\theta=8$ is needed for molecular weights and fraction of sol. Such simulations are crucial for the operation of the reactors and are essential for a correct interpretation of the properties of the polymer obtained. Similar results are presented in Figure 3 for $\tau=12 \mathrm{~h}$.

The comparison between weight average molecular weights in a CSTR with different values of space-time is done in Figure 4. For $\tau=2$ and $4 \mathrm{~h}$ there is no gelation and up to the critical value, the final average molecular weights increase with the space-time. For values of $\tau$ higher than its critical value, in the steady state the average molecular weight of sol decreases when space-time increases. A similar plot is shown in Figure 5 for the weight fraction of sol. The final weight fraction of sol decreases by increasing

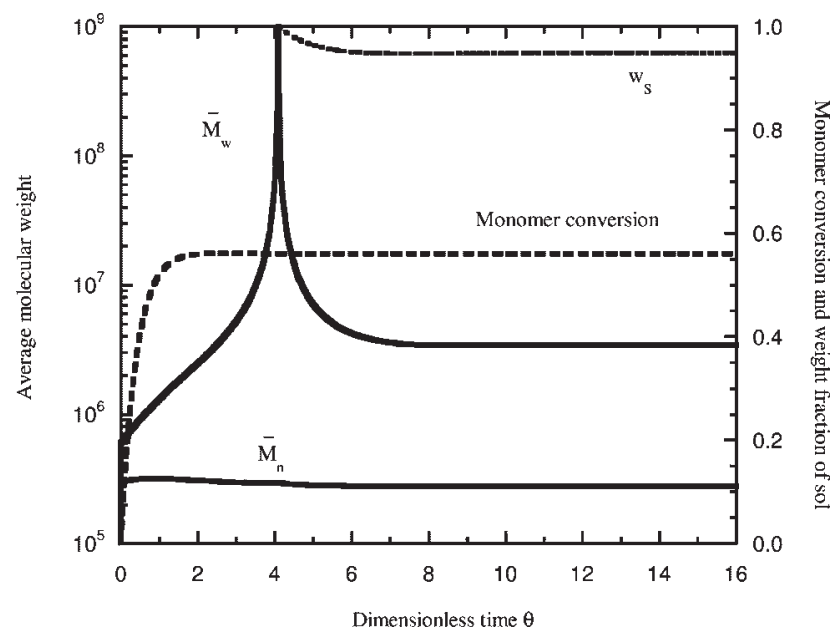

Figure 2. Unsteady state operation of a CSTR with $\tau=8 \mathrm{~h}$.

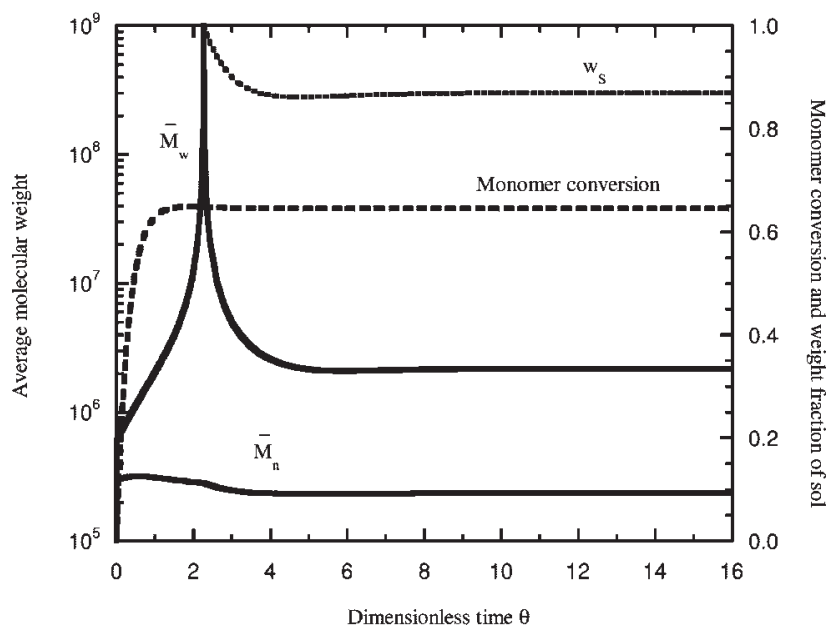

Figure 3. Unsteady state operation of a CSTR with $\tau=12 \mathrm{~h}$.

$\tau$ and also attains slowly a steady state, with an unexpected inflection point in its time evolution.

\section{Comparison With Alternative Methods}

When a new method for simulation is presented the natural question that always arises is: what are the advantages and the differences relatively to other methods? Comparison with experimental data is obviously a very important test. As presented along the text, the greatest advantage of this method is the possibility of disregarding some mathematical approximations, which need no longer to be checked, and thus obtain more reliable results. This is possible due to a more complex mathematical formulation, which has the drawback of also increasing computing time, and for some applications (on line monitoring and/or control in the postgel region) this can constitute a shortcoming of the method

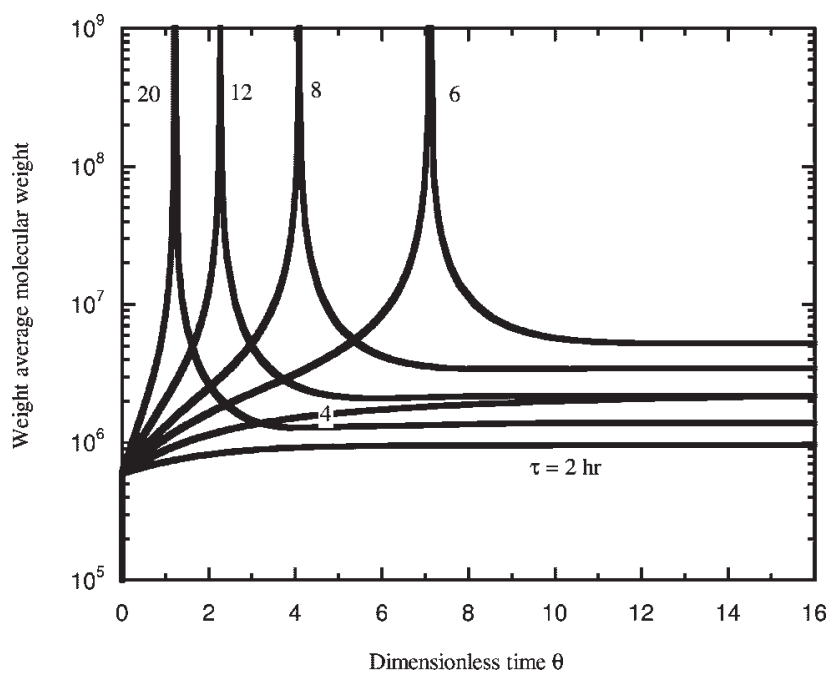

Figure 4. Weight average molecular weight for unsteady state operation of a CSTR with different values of space-time $(\tau)$. 


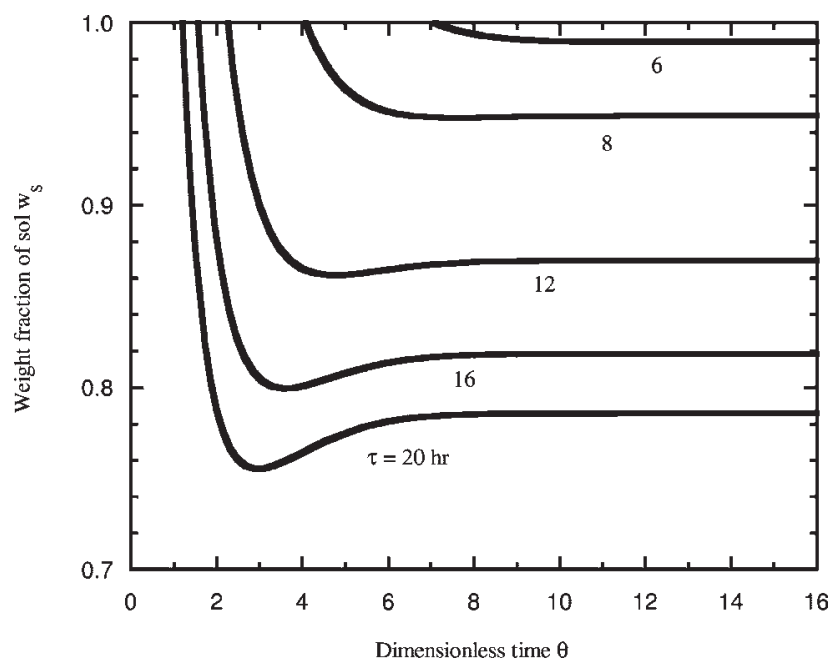

Figure 5. Weight fraction of sol for unsteady state operation of a CSTR with different values of space-time $(\tau)$.

in its present form. Nevertheless, we think that even so, the existence of a rigorous tool is always important, namely for detecting possible failures of approximated methods in describing real systems.

The objective of this section is, as far as possible, to discuss and compare the predictions of this approach with others which have been previously used. In reality, a full comparison of all results is not an easy task because, as far we know, simulations of exactly the same kinetic scheme with other methods have never been carried out, just subsets of it, for CSTRs with or without gelation. It is possible to find in the literature works which study pre-gelation behavior of continuous reactors ${ }^{[45]}$ and the corresponding extension to the post-gelation regime by using the "numerical fractionation" technique for transient state. ${ }^{[46]}$ Nevertheless, in these works, the analyzed system was the chain coupling of polymers (a non-linear poly-condensation with a single functional group). This is a completely different (and much simpler) system and a comparison with this work is not relevant. Other methods were applied for the simulation of long-chain branching or vinyl/divinyl copolymerization in continuous free radical polymerizations: Monte Carlo simulation, ${ }^{[25]}$ but retaining the steady state, "numerical fractionation" technique ${ }^{[23,47]}$ without transient behavior analysis and Galerkin finite-element method $^{[26]}$ but without quantifying the amount of gel. This has been more recently taken into account by excluding from sol the polymer molecules with some high enough degree of polymerization. ${ }^{[28]}$

In order to make possible some fair comparisons with the results obtained in this work, the steady state predictions that are a result of the transient calculations will be used. For a first case study, it will be used a system similar to a continuous polymerization of vinyl acetate which is a classical problem where some simplified versions of the method of the moments may fail to obtain a realistic description.
Additionally, a great number of experimental data for vinyl acetate polymerization are available in literature. In fact, for this system the use of inappropriate models, namely the lack of consideration of the existence of poly-radicals and neglect of radical outflow can lead to the divergence of the second moment of CLD and therefore to the prediction of gelation contrary to experimental evidence, as stated by Tobita. ${ }^{[2]}$ The experimental data and chosen operation conditions can be found in works by Chatterjee et al. ${ }^{[15,16]}$ namely with $y_{\mathrm{S}}=2, C_{\mathrm{M}}=2.46 \times 10^{-4}, C_{\mathrm{P}}=1.30 \times 10^{-4}$, $C_{\mathrm{S}}=0.34 \times 10^{-4}$, and $K_{2}=0.66$, which are also the same parameters used by Tobita with the goal of comparison with his Monte Carlo simulations. ${ }^{[25]}$ In Figure 6, comparisons between these experimental results and the predictions of two versions of the method of the moments, Monte Carlo simulation, and the present method are presented. The predictions of method of moments 1 were obtained according to Chatterjee et al. ${ }^{[15,16]}$ and method of moments 2 is based on an improved version of this method. ${ }^{[21]}$ The inability of both methods of moments to calculate the second order (or higher) moment of CLD is clear. Divergence predicted for the second order moment (gelation) is not experimentally observed, and therefore a realistic description of this system is not obtained. The method of moments 2 presents a good agreement with experimental data for low steady state monomer conversion but for high conversions, the description of the system fails, in our opinion, due to the set of simplifying assumptions used.

A much better prediction is obtained by Monte Carlo simulation. This is a consequence of considering the existence of poly-radicals and taking into account radical outflow. ${ }^{[25]}$ Results obtained by the method here described present an excellent agreement with the experimental data, specially taking into account that no fitting of rate parameters was done. The improved results obtained by this

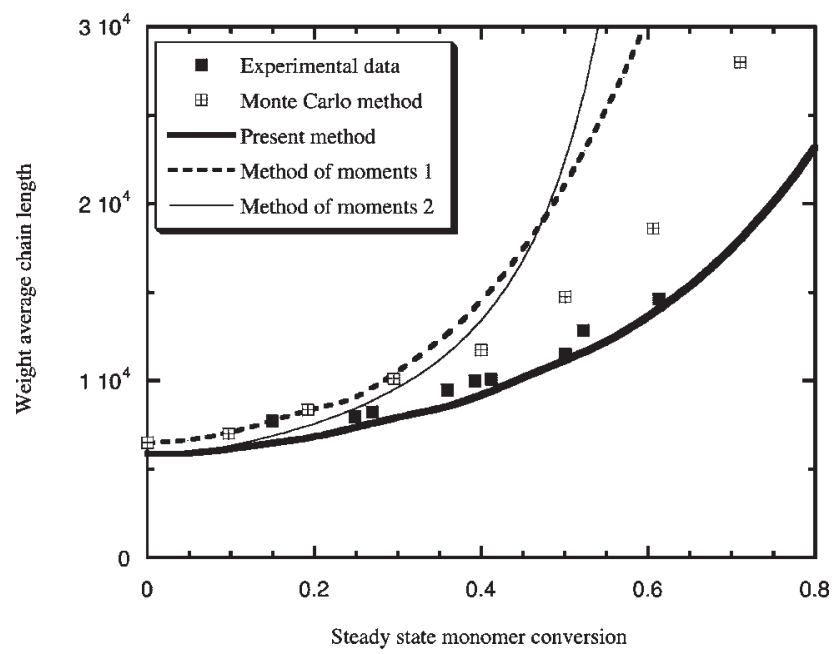

Figure 6. Comparison between different simulation methods and experimental data according to Chatterjee et al. ${ }^{[15,16]}$ for polymerization of vinyl acetate in a CSTR. 
method comparatively to the Monte Carlo simulation are, in our opinion, explained by the elimination of the assumption of negligible initiation and termination reaction rates relatively to propagation and transfer. This fact confirms the suspicion that a more rigorous model is essential in the description of non-linear free radical polymerizations, and that in some circumstances appreciable discrepancies are observed between the prediction of different models, as stated before for other kind of systems. ${ }^{[35]}$

The numerical fractionation technique is based on the method of the moments because this method is used and applied to each generation individually. ${ }^{[22]}$ One of the main advantages of numerical fractionation relatively to the method of moments is the possibility to extend the calculations to the post-gelation regime in a simple way. Nevertheless, the set of equations used with numerical fractionation inherits the approximations usually done by the method of moments. Population balances can be derived with variable degrees of complexity ${ }^{[21,48]}$ but some level of approximation has always been retained. Quasi-steady state approximation, neglecting poly-radicals, and closure conditions are examples of approximating conditions that can be found in published works where numerical fractionation technique or simplified versions of the method of the moments have been used. ${ }^{[22]}$ When these methods are extended to the analysis of $\mathrm{CSTR}^{[23]}$ additional approximation conditions like neglecting outflow of radicals are introduced.

In order to make a comparison between the predictions of the present method with published related works of numerical fractionation technique, some simplification in the kinetic scheme (see Table 3) is inevitable: in published works where this kind of polymerization systems is analyzed by numerical fractionation ${ }^{[22,23]}$ only transfer to polymer is considered as branching mechanism. Therefore, we had to suppress terminal double bonds propagation from the kinetic scheme; this does not mean that numerical fractionation could not take into account that reaction with a supplementary effort, which is not really worth doing just for the sake of a comparison of very basic prediction capabilities as it is done in this work. In order to isolate the effect of chain transfer to polymer, chain transfer to monomer and chain transfer to solvent were also left out of consideration. The kinetic parameters needed for these simulations are obtained setting equal to zero all unnecessary parameters in Table 4. For these reaction conditions, the predictions of numerical fractionation technique have been obtained following the principles presented in literature. ${ }^{[22,23]}$ Saidel and Katz approximation for the third moment, ${ }^{[49]}$ was used as closure condition.

It was stated ${ }^{[23]}$ that the closure assumption has no effect on the prediction of the gel point or the molecular weight averages, only on CLD. The number of generations in the calculations has been varied between 8 and 30, even if the authors ${ }^{[22]}$ have stated that a number of 8 to 10 should be

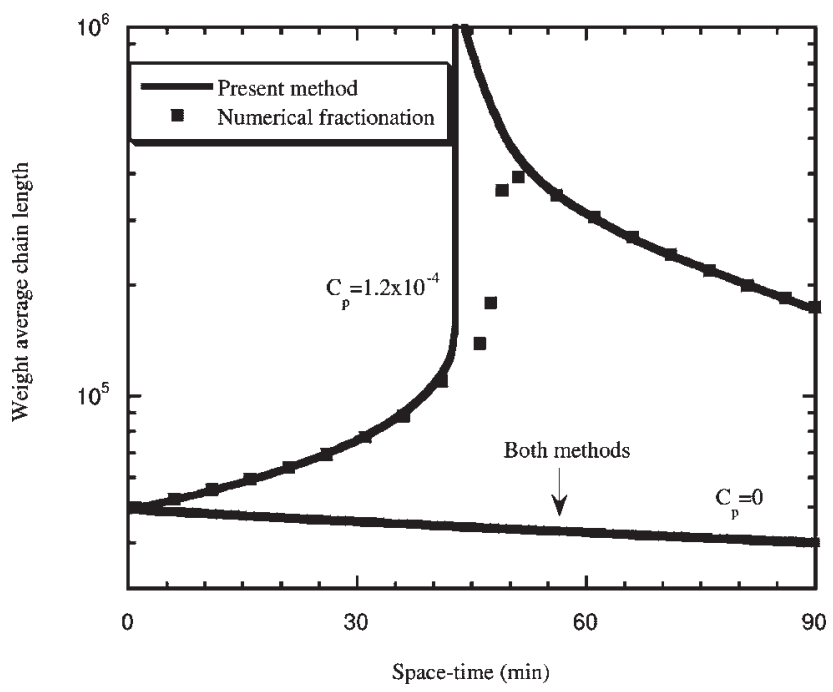

Figure 7. Steady state predictions of the numerical fractionating technique and present method for a CSTR when transfer to polymer is the only branching mechanism.

enough in practice. Our coding of numerical fractionation has been checked in different ways, namely, by reproducing the results of non-linear polymerization in batch reactor as published in ref. ${ }^{[22]}$ The weight average chain length for the linear case is also consistent between the two techniques and the same is observed for the number average chain length in the linear and non-linear cases.

In Figure 7 are presented the predictions of the two methods for the steady state weight average chain length of the resulting polymers. To test the consistency of the methods the linear case $\left(C_{\mathrm{P}}=0\right)$ was also simulated, and no significant difference is observed. Nevertheless, in the nonlinear system $\left(C_{\mathrm{P}}=1.2 \times 10^{-4}\right)$ the differences between the predictions become very important in the neighborhood of the critical space-time of gelation.

It is shown in Figure 8 that the predictions of numerical fractionation do not improve on increasing the number of generations. Since our calculations compute all moments with respect to the different chain lengths, the weight average number of radicals per molecule $\frac{\sum_{a_{1}=0}^{\infty} \cdots \sum_{a_{4}=0}^{\infty} a_{1}^{2} \mathrm{P}(\mathbf{a})}{\sum_{a_{1}=0}^{\infty} \cdots \sum_{a_{4}=0}^{\infty} a_{1} \mathrm{P}(\mathbf{a})}$ is a useful by-product as it can detect the presence of multiradicals. In fact, that quantity is nearly always close to one, presenting very sharp maxima around gel point. Table 6 shows this behavior in detail. This result simply confirms previous statements about the existence of polyradicals, which is known to be important in the vicinity of gelation. ${ }^{[50,51]}$ As we are considering an ideal CSTR, the outflow composition is the same inside the reactor. Neglecting radical outflow, in spite of its low concentration, is obviously an approximation with an effect becoming more pronounced as the space-time becomes smaller. The observed discrepancies between the exact results of the present method and numerical fractionation are then a result 


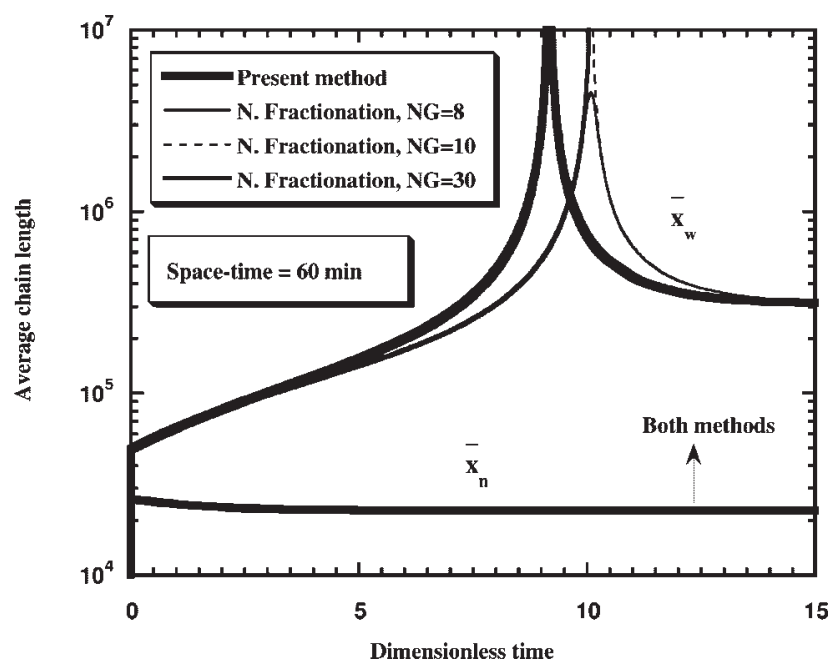

Figure 8. Comparison of the transient predictions of average chain length for a free radical polymerization with transfer to polymer in a CSTR with $\tau=60$ min using the present method and numerical fractionation with different number of generations.

of the various approximations which are inherent to the latter technique. It is plausible that differences between the two methods may become even larger when more complex kinetic schemes are involved, namely in the presence of terminal double bond propagation or other mechanisms of branching.

\section{Prediction of Chain Length Distributions}

In this section, it is shown that the present method can be also used to predict CLDs. In order to keep this presentation within a manageable size, only the main steps necessary for its numerical implementation are discussed.

The "natural" way to perform the calculation of the CLD is by using Cauchy's integral formula to carry out the inversion of the moment generating function, $G(s)$ to obtain $\mathrm{P}(n)$ with $n=0,1 \ldots, \infty$. As $G(s)$ is only numerically available, the evaluation of the CLD is based on the discretization of the Cauchy's integral. This approach has been used in the past to obtain CLD with poly-condensations and some non-radical poly-additions. ${ }^{[33,52,53]}$ An analysis of its truncation and round-off errors ${ }^{[54]}$ shows this is a reliable method. Still, when average chain length increases,

Table 6. Some values of the weight average number of polymer radicals in the vicinity of the gel point in a CSTR with $\tau=60 \mathrm{~min}$.

Dimensionless time $\left(\theta=\frac{t}{\tau}\right) \quad$ Weight average number of polymer radicals

9.17176373

9.17267109

9.17274422

9.17275906

9.17276089

9.17276095

9.17341346 it becomes too slow, as it requires as many values of the transform or generating function as the upper value of chain length where distribution is to be evaluated. A better algorithm is needed to deal with that situation. It has been suggested ${ }^{[54]}$ that determination of asymptotic behavior of the distribution should be tried. This would require finding the singularities of the generating function closest to the origin, or Hayman's method ${ }^{[55]}$ in case they do not exist. The application of these ideas with numerically estimated generating functions has not yet been tried.

The practical implementation of the aforementioned method requires the evaluation of $G(s)$ in the complex domain and therefore the characteristics must be also integrated in the same domain. In order to perform this evaluation the generic equations of the present method ${ }^{[37]}$ have also been extended to the complex domain. At this point, it should be stated that the integration of the characteristics of thousands of different final values of the complex argument $\left(s_{f}\right)$ discourage even more the application of this method to invert $G(s)$. In fact, with free radical polymerizations, due to the stiffness of the differential equations involved and the oscillatory behavior of the characteristics in the complex domain, its numerical integration is very slow and the prediction of CLD by using this approach was found to be impracticable.

In recent years, some researchers have suggested the use of Laplace transforms, the continuous counterpart of discrete moment generating functions or $z$-transforms, in order to predict CLD. After Miller et al. ${ }^{[56]}$ have shown the potential of this method, Sarmoria ${ }^{[57-61]}$ and collaborators have been using it in process modeling of several polymerization systems. In this latter series of papers, a general framework for the application of probability generating functions to mass balances was presented, a few inversion algorithms were tested, and their validation was performed by comparison with experimental results.

There is some convergence of the above described approach with the methods described in this paper. Nevertheless, considerable differences in the mathematical treatments to evaluate the moment generating function can be identified. In fact, with the present method the evaluation of $G(s)$ is free from approximation conditions like closure conditions for the moments which have been used in the aforementioned works. Other important advantage of this method is the fact that it can be used for the prediction of CLD when gelation occurs. These differences do not invalidate the use of the proposed algorithms to recover the CLD, and improved predictions are expected due to the higher accuracy in the evaluation of $G(s)$. Indeed, on trying to obtain the CLDs next presented, we could confirm the good foundation of previous guidelines for the proper use of inversion methods. ${ }^{[59]}$ Following the recommendation that the inversion results should be checked by using at least two different algorithms, Papoulis ${ }^{[62]}$ and Weeks ${ }^{[63]}$ inversion methods have been implemented. 


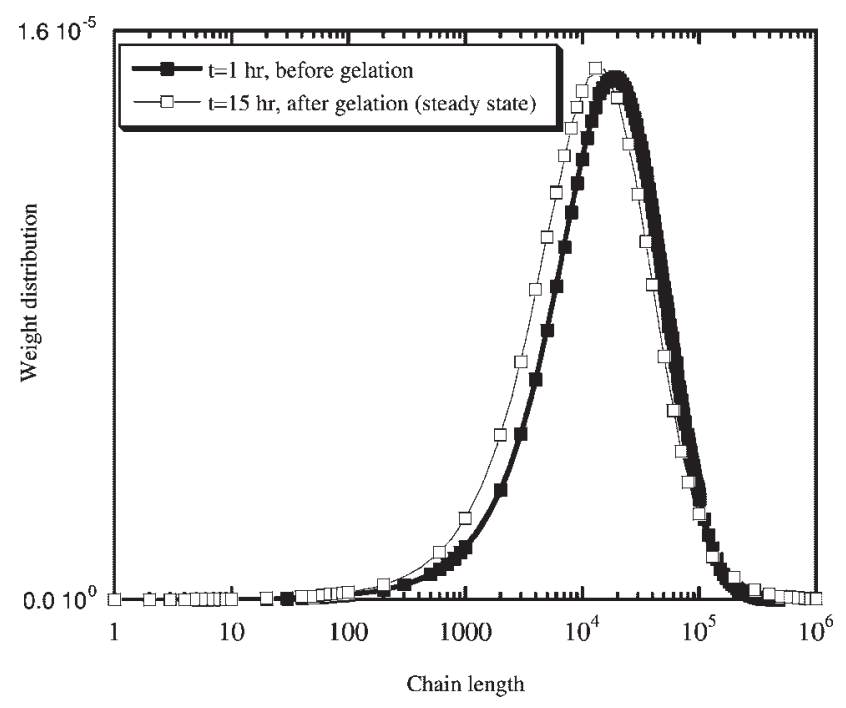

Figure 9. Predictions of the chain length distribution (CLD) before $(t=1 \mathrm{~h})$ and after gelation $(t=15 \mathrm{~h}$, steady state) for a free radical polymerization with transfer to polymer in a CSTR with $\tau=60 \mathrm{~min}$.

In Figure 9 are presented the predictions of the weight CLD for the transient behavior of a CSTR with $\tau=60 \mathrm{~min}$, from which average properties had been previously shown in Figure 8. It is therefore possible with the present method to predict CLD before gelation $(t=1 \mathrm{~h})$ and also after gelation ( $t=15 \mathrm{~h}$, steady state). In Figure $10 \mathrm{a} \log -\log$ plot of the CLDs for $t=1$ and $15 \mathrm{~h}$ and near the gel point is presented. With this representation, it is clear that the main difference between the distributions occurs in the end tail corresponding to high degrees of polymerization. Numerical inversion can be performed using different

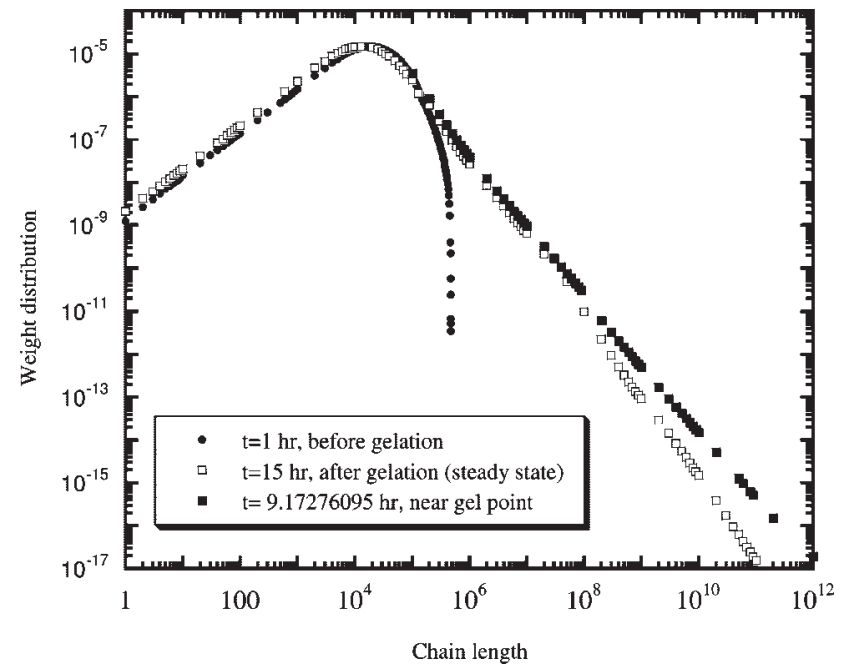

Figure 10. A log-log plot of the predicted CLD before gelation $(t=1 \mathrm{~h})$, after gelation ( $t=15 \mathrm{~h}$, steady state) and near the gel point for a free radical polymerization with transfer to polymer in a CSTR with $\tau=60 \mathrm{~min}$ transformed quantities, such as $G(s)=\sum_{i=0}^{\infty} s^{i} \mathrm{P}(i)$, $G_{w}(s)=\sum_{i=0}^{\infty} s^{i} \mathrm{P}(i)$, and $G_{z}(s)=\sum_{i=0}^{\infty} s^{i} i^{2} \mathrm{P}(i)$. In the description of the end tail of the distributions, a better numerical performance is obtained by using $G_{z}(s)$ to execute the numerical inversion. This behavior can be explained through the high sensitivity of $G_{z}(s)$ to variations in $s$ when $\mathrm{P}(i)$ is very low.

The cumulative distributions, $C(n)=\sum_{i=0}^{n} \mathrm{P}(i), C_{w}(n)=$ $\sum_{i=0}^{n} i \mathrm{P}(i)$, and $C_{z}(n)=\sum_{i=0}^{n} i^{2} \mathrm{P}(i)$ can also be obtained by numerical inversion using the same principles before presented to compute the CLD. Prediction of these distributions can be used to obtain an estimate of a global error in the numerical inversion of the CLD, which can be conveniently defined as $\varepsilon=1-\frac{\sum_{i=0}^{n_{\max }} \mathrm{P}(i)}{\sum_{i=0}^{\infty} \mathrm{P}(i)}$. Similar definitions can be introduced with $C_{w}(n)$ and $C_{z}(n)$. By choosing an upper bound to the degree of polymerization $\left(n_{\max }\right)$, the corresponding cumulative distribution $\left[C\left(n_{\max }\right)\right]$ is obtained by numerical inversion and is compared with the zero moment of the distribution $\left(\sum_{i=0}^{\infty} \mathrm{P}(i)\right)$ which can be computed independently from the inversion process. Similar calculations can be performed with the first and second moments of the distribution. In the distributions presented in this work, an upper value of error $\varepsilon<10^{-3}$ has always been achieved.

In Figure 11 the weight distribution of the number of radicals near the gel point for the same system is shown to present a nearly exponential shape. This distribution is defined by $\frac{r \mathrm{P}(r)}{R}$ where $\mathrm{P}(r)$ is the polymer concentration with $r$ radicals and $R$ is the total macro-radicals concentration. The weight average of the number of radicals near the gel point had already been presented in Table 6 and the importance of the presence of poly-radicals near gelation is confirmed once again.

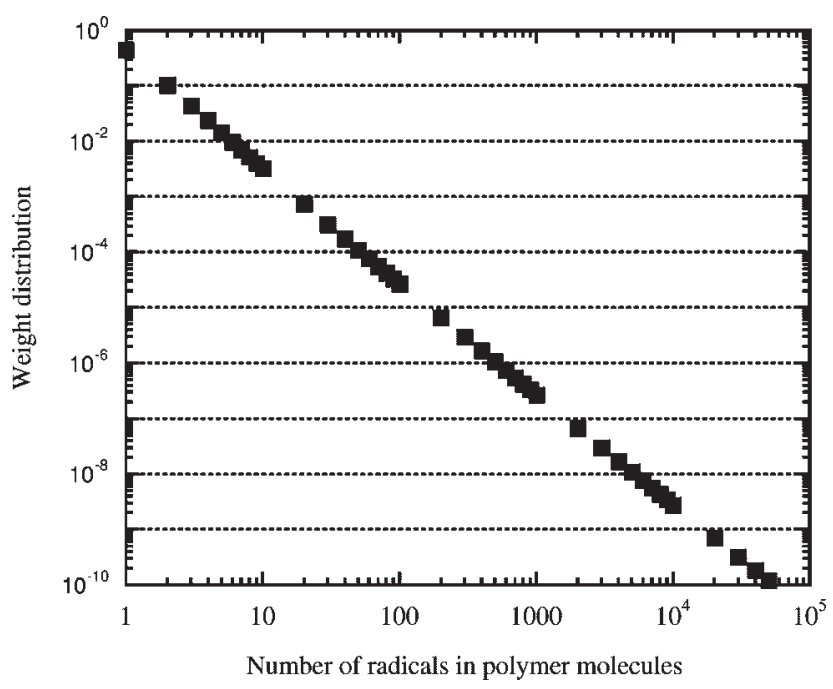

Figure 11. Prediction of the weight distribution of number of radicals near the gel point for a free radical polymerization with transfer to polymer in a CSTR with $\tau=60 \mathrm{~min}$. 


\section{Conclusion}

A general model for irreversible non-linear polymerizations was applied to the study of terminal branching and chain transfer to polymer in CSTR, when homogeneous kinetics (bulk/solution and many suspension processes) is valid.

This method can deal with complex chemical systems, before and after gelation, without sacrificing mathematical exactness. As it is based upon a kind of interpreter of irreversible polymerization schemes, it avoids the timeconsuming and error-prone direct manipulation of population balance equations.

The present approach is expected to become a useful tool for the analysis of non-linear free radical polymerizations in continuous reactors, namely:

- For improving the level of knowledge and control, in preand post-gel regions, of the polymer produced in this kind of reactors.

- For selecting the appropriate operation conditions in order to prevent gelation if desired.

A key concept in the treatment of non-linear free radical polymerizations here presented is the lack of distinction between "active" and "dead" polymer molecules, which allows a cleaner mathematical description, without several simplifications with non-universal applicability which have been introduced in the past in order to obtain mathematically tractable models. For CSTR predictions these kind of approximations can have important effects and some unrealistic results may be obtained. For instance, the method of the moments sometimes predicts gelation in systems where it does not occur. Also, many of these methods are unable to deal with the presence of gel.

Other recent proposed methods, like Galerkin finiteelements method, have interesting potential use in predicting CLD for simpler kinetic schemes leading to $1 \mathrm{D}$ distributions. Some limitations of commercial software using this method could be overcome ${ }^{[28]}$ and an analysis of free radical polymerization with transfer to polymer, also in the presence of gel, has been presented. This approach is an alternative to the Laplace domain method discussed in this paper, becoming particularly competitive when dealing with chain length dependent reactivity (as happens with free radical termination in many situations).

Due to the need to avoid a high computational effort when very fast and slow chemical reactions are simultaneously present, implementation of Monte Carlo simulation of these systems is not straightforward and simplifying approximations so far introduced in order to obtain practical results have been deleterious to agreement with experimental data.

Numerical fractionation has been developed to model non-linear polymerization processes, possibly with gel formation. Discontinuities at the gel point are avoided and predictions in the presence of gel can be carried out in a computationally fast way. Nevertheless, it has used simplified versions of the method of the moments and therefore it has been affected by their inherent lack of exactness. Thus, the quality of its predictions deteriorates when molecules with several radicals are present.

The most remarkable qualitative results of the new simulations presented in current work are the dynamics of change of average molecular weights, which show much longer times to reach steady state than monomer conversion.

The computational performance of the current technique is enough for predictions of average molecular weights in the absence of gel (about $50 \mathrm{~ms}$ per point for the chemical systems here considered), but a lot of improvement is needed in the presence of gel.

It is finally confirmed the usefulness of this approach to predict CLD by taking advantage of numerical methods widely used for Laplace transform inversion.

[1] Y. Lu, J. A. Biesenberger, Polym. Eng. Sci. 1997, 37, 1036.

[2] P. J. Flory, "Principles of Polymer Chemistry", Cornell University Press, Ithaca, NY 1953, Chapter 9.

[3] G. Odian, "Principles of Polymerization", $3^{\text {rd }}$ edition, Wiley-Interscience, New York 1991, pp. 255-259.

[4] G. Moad, D. H. Solomon, "The Chemistry of Free Radical Polymerization", Pergamon Press, Oxford, UK 1995.

[5] C. H. Bamford, E. F. T. White, Trans. Faraday Soc. 1958, 54 , 278.

[6] J. Mikes, K. Dušek, Macromolecules 1982, 15, 93.

[7] C. H. Bamford, H. Tompa, Trans. Faraday Soc. 1954, 50, 1097.

[8] K. Nagasubramanian, W. W. Graessley, Chem. Eng. Sci. 1970, 25, 1549.

[9] D. J. Stein, Makromol. Chem. 1964, 76, 170.

[10] W. W. Graessley, H. Mittelhauser, Makromol. Chem. 1965, $86,129$.

[11] W. W. Graessley, H. Mittelhauser, J. Polym. Sci. A2 1967, 5, 431.

[12] W. W. Graessley, R. D. Hartung, W. C. Uy, J. Polym. Sci. A2 1969, 7, 1919.

[13] K. Nagasubramanian, W. W. Graessley, Chem. Eng. Sci. 1970, 25, 1559.

[14] J. C. Hyun, W. W. Graessley, S. G. Bankoff, Chem. Eng. Sci. 1976, 31, 945.

[15] A. Chatterjee, K. Kabra, W. W. Graessley, J. Appl. Polym. Sci. 1977, 21, 1751.

[16] A. Chatterjee, W. S. Park, W. W. Graessley, Chem. Eng. Sci. 1977, 32, 167.

[17] W. Baade, H. U. Moritz, K. H. Reichert, J. Appl. Polym. Sci. 1982, 27, 2249.

[18] T. W. Taylor, K. H. Reichert, J. Appl. Polym. Sci. 1985, 30, 227.

[19] D. J. Arriola, Ph.D. thesis, University of Wisconsin, USA 1989.

[20] R. A. Hutchinson, Polym. React. Eng. 1993, 1, 521.

[21] A. Baltsas, D. S. Achilias, C. Kiparissides, Macromol. Theory Simul. 1996, 5, 477. 
[22] F. Teymour, J. D. Campbell, Macromolecules 1994, 27, 2460.

[23] G. Papavasiliou, I. Birol, F. Teymour, Macromol. Theory Simul. 2002, 11, 533

[24] H. Tobita, J. Polym. Sci., Part B: Polym. Phys. 1994, 32, 901.

[25] H. Tobita, J. Polym. Sci., Part B: Polym. Phys. 1994, 32, 911.

[26] P. D. Iedema, H. C. Hoefsloot, Macromol. Theory Simul. 2002, 11, 410 .

[27] P. D. Iedema, S. Grcev, H. C. Hoefsloot, Macromolecules 2003, 36, 458 .

[28] P. D. Iedema, H. C. Hoefsloot, Macromolecules 2004, 37, 10155.

[29] U. Budde, M. Wulkow, Chem. Eng. Sci. 1991, 46, 497.

[30] M. Wulkow, Macromol. Theory Simul. 1996, 5, 393.

[31] S. I. Kuchanov, L. M. Pis'men, Vysokomol. Soyed. 1971, A13, 2035.

[32] M. R. P. F. N. Costa, R. C. S. Dias, Chem. Eng. Sci. 1994, 49, 491.

[33] M. R. P. F. N. Costa, R. C. S. Dias, DECHEMA Monogr $1995,131,523$.

[34] M. R. P. F. N. Costa, R. C. S. Dias, DECHEMA Monogr. $1998,134,197$.

[35] M. R. P. F. N. Costa, R. C. S. Dias, Macromol. Theory Simul. 2003, 12,560

[36] R. C. S. Dias, M. R. P. F. N. Costa, Macromolecules 2003, 36 , 8853.

[37] M. R. P. F. N. Costa, R. C. S. Dias, Chem. Eng. Sci. 2005, 60, 423.

[38] P. Cacioli, D. G. Hawthorne, R. L. Laslett, R. E. Rizzardo, D. H. Solomon, J. Macromol. Sci., Chem. 1986, A23, 839.

[39] K. Miyake, P. B. Zetterlund, B. Yamada, Macromol. Rapid Commun. 2004, 25, 1905.

[40] N. Ahmad, F. Heatley, P. A. Lovell, Macromolecules 1998, $31,2822$.

[41] D. Britton, F. Heatley, P. A. Lovell, Macromolecules 1998 , 31,2828 .
[42] K. A. Hutchinson, J. R. Richards, M. T. Aronson, Macromolecules 1994, 27, 4530.

[43] E. Hairer, G. Wanner, "Solving Ordinary Differential Equations II", $2^{\text {nd }}$ revised edition, Springer, Berlin 2002, pp. 566-574.

[44] J. R. Cash, G. Moore, R. W. Wright, ACM Trans. Math. Soft. 2001, 27, 245.

[45] C. Cozewith, W. W. Graessley, G. Ver Strate, Chem. Eng. Sci. 1979, 34, 245.

[46] C. Cozewith, F. Teymour, AIChE J. 1998, 44, 722.

[47] G. Papavasiliou, F. Teymour, Macromol. Theory Simul. 2003, 12, 543.

[48] P. Pladis, C. Kiparissides, Chem. Eng. Sci. 1998, 53, 3315.

[49] G. M. Saidel, S. Katz, J. Polym. Sci. A2 1968, 5, 1149.

[50] S. Zhu, Macromolecules 1996, 29, 456.

[51] H. Tobita, S. Zhu, J. Polym. Sci., Part B: Polym. Phys. 1996, 34, 2099.

[52] P. L. Mills, Comput. Chem. Eng. 1986, 10, 399.

[53] M. R. P. F. N. Costa, J. Villermaux, Ind. Eng. Chem. Res. 1988, 27, 421.

[54] J. Abate, W. Whitt, Oper. Res. Lett. 1992, 12, 245.

[55] W. Hayman, J. Reine Angew. Math. 1956, 196, 67.

[56] N. C. Miller, R. W. Toffolo, K. B. McAuley, P. J. McLellan, Polym. React. Eng. 1996, 4, 279.

[57] M. Asteasuain, C. Sarmoria, A. Brandolin, Polymer 2002, 43, 2363.

[58] M. Asteasuain, C. Sarmoria, A. Brandolin, Polymer 2002, 43, 2513.

[59] M. Asteasuain, A. Brandolin, C. Sarmoria, Polymer 2002, $43,2529$.

[60] M. Asteasuain, C. Sarmoria, A. Brandolin, J. Appl. Polym. Sci. 2003, 88, 1676.

[61] M. Asteasuain, C. Sarmoria, A. Brandolin, Polymer 2004, $45,321$.

[62] A. Papoulis, Quart. Appl. Math. 1956, 14, 405.

[63] W. T. Weeks, J. Assoc. Comp. Mach. 1966, 13, 419. 